\title{
Personality and Recommender Systems
}

\author{
Marko Tkalčič and Li Chen
}

\begin{abstract}
Personality, as defined in psychology, accounts for the individual differences in users' preferences and behaviour. It has been found that there are significant correlations between personality and users' characteristics that are traditionally used by recommender systems ( e.g. music preferences, social media behaviour, learning styles etc.). Among the many models of personality, the Five Factor Model (FFM) appears suitable for usage in recommender systems as it can be quantitatively measured (i.e. numerical values for each of the factors, namely, openness, conscientiousness, extraversion, agreeableness and neuroticism). The acquisition of the personality factors for an observed user can be done explicitly through questionnaires or implicitly using machine learning techniques with features extracted from social media streams or mobile phone call logs. There are, although limited, a number of available datasets to use in offline recommender systems experiment. Studies have shown that personality was successful at tackling the cold-start problem, making group recommendations, addressing cross-domain preferences 4 and at generating diverse recommendations. However, a number of challenges still remain.
\end{abstract}

\section{Introduction}

In recent years, there has been an increased research interest in more user-oriented approaches in recommender systems, where various psychological aspects have been investigated (e.g. personality [28] and emotions [70]) compared to the classical machine-learning approaches in recommender systems (i.e. classical ratings

Marko Tkalčič

Johannes Kepler University, Altenberger Strasse 69, Linz, Austria, e-mail: marko.tkalcic@ jku.at

Li Chen

Hong Kong Baptist University, 224 Waterloo Road, Kowloon Tong, Kowloon, Hong Kong, e-mail: lichen@ comp.hkbu.edu.hk 
prediction from the user-item matrix, such as the Netflix-prize problem [36]). As argued in Ch. ??, an important function of recommender systems is to help people make better decisions. As personality plays an important role in decision-making [13] it should be taken into account. It has been also argued that an improvement in the rating prediction accuracy (usually measured with measures such as the Root Mean Square Error, see also Ch. ??) does not necessarily mean a better user experience [45]. As further discussed in Ch. ??, assessing the recommender systems from a user-centric perspective yields a better picture of the quality of the recommender system under study. Hence, when optimizing a recommender system for user-centric aspects one should take into consideration these aspects already in the design of the recommender systems. This is why personality, which by definition measures individual users' differences [33], should be taken into account in order for the recommender system to perform better in user-centric metrics.

The individual differences between users, as described by personality, are useful in a wide range of aspects of recommender systems. For example, music preferences have been shown to correlate with personality [55]. It has been shown that people with different personalities can be more or less inclined to consume novel items, so the degree of diversity in presenting recommended items can be personalized accordingly [74]. Personality has been used to improve user-similarity calculation in the new-user problem $[69,29]$. Also, group modeling based on personality has improved the performance of group recommendations [56, 54, 35].

In this chapter we present personality-based recommender systems. We focus on the tools needed to design such systems, especially on (i) personality acquisition methods and (ii) strategies for using personality in recommender systems.

From its definition in psychology, personality accounts for the individual differences in our enduring emotional, interpersonal, experiential, attitudinal and motivational styles [33]. Incorporating these differences in the recommender system appears to be a natural choice for delivering personalized recommendations. Furthermore, personality parameters can be quantified as feature vectors, which makes them suitable to use in computer algorithms. However, the acquisition of personality parameters for individual users could be, until recently, acquired only through extensive questionnaires, which was an obstacle in a day-to-day use of recommender systems. Examples of such questionnaires are the International Personality Item Pool (IPIP) [23] and the NEO Personality Inventory [43]. Recently, several investigations have been conducted to extract personality parameters in an implicit way from social media streams [37, 53, 22]. Valuable sources for assessing the personality of a user without bothering her/him with extensive questionnaires are social media streams (e.g. Facebook [37], blogs [32] or Twitter [53]) and other user-generated data streams (e.g. email [63]).

The chapter is organized as follows. In Sec. 2 we survey various models of personality that were developed and are suitable for recommender systems. In Sec. 3 we present various methods for acquiring personality, which fall in either of the two categories: implicit or explicit. In Sec. 4 we discuss various strategies that exploit personality and have been used so far in recommender systems. Further, in Sec. 5 
we present the challenges that are still ahead in the domain of personality-based recommender systems. Finally we provide some conclusive thoughts in Sec. 6

\section{What is personality?}

According to [44], personality accounts for the most important ways in which individuals differ in their enduring emotional, interpersonal, experiential, attitudinal and motivational styles. Translated into the recommender systems terminology, personality can be thought of as a user profile, which is context-independent (it does not change with time, location or some other context - see Ch. ?? for context in recommender systems) and domain-independent (it does not change through different domains, e.g. books, movies - see also Ch. ?? for personality in cross-domain recommender systems).

Historically, the first reports of studies of individual differences among humans go back to the ancient Greeks with the Hippocrates' Four Humours that eventually led to the personality theory known today as the four temperaments (Choleric, Sanguinic, Melancholic and Phlegmatic) [34].

Today, the Five Factor Model of personality (FFM) [44], is considered one of the most comprehensive and is the mostly used personality model in recommender systems $[72,28,29,30,10,74,67,18,48,49]$. The FFM is sometimes referred to also as the Big-Five (Big5) model of personality.

\subsection{The Five Factor Model of Personality}

The roots of the FFM lie in the lexical hypothesis, which states that things that are most important in people's lives eventually become part of their language. Studying the usage of language, researchers extracted a set of adjectives that describe permanent traits (see Tab. 1). With further research, these adjectives were clustered into the five main dimensions: openness to experience, conscientiousness, extraversion, agreeableness, and neuroticism (the acronym OCEAN is often used) [44].

\begin{tabular}{ll}
\hline Factor & Adjectives \\
\hline Extraversion (E) & active, assertive, energetic, enthusiastic, outgoing, talkative \\
Agreeableness (A) & appreciateive, forgiving, generous, kind, sympathetic, trusting \\
Conscientiousness (C) & efficient, organized, planful, reliable, responsible, thorough \\
Neuroticism (N) & anxious, self-pitying, tense, touchy, unstable, worrying \\
Openness (O) & artistic, curious, imaginative, insightful, original, wide interest \\
\hline
\end{tabular}

Table 1: Examples of adjectives related to the FFM [44] 
Openness to Experience (O), often referred to just as Openness, describes the distinction between imaginative, creative people and down-to-earth, conventional people. High $\mathrm{O}$ scorers are typically individualistic, non conforming and are very aware of their feelings. They can easily think in abstraction. People with low $\mathrm{O}$ values tend to have common interests. They prefer simple and straightforward thinking over complex, ambiguous and subtle. The sub-factors are imagination, artistic interest, emotionality, adventurousness, intellect and liberalism.

Conscientiousness (C) concerns the way in which we control, regulate and direct our impulses. People with high $\mathrm{C}$ values tend to be prudent while those with low values tend to be impulsive. The sub-factors are self-efficacy, orderliness, dutifulness, achievement-striving, self-discipline and cautiousness.

Extraversion (E) tells the degree of engagement with the external world (in case of high values) or the lack of it (low values). The sub-factors of $E$ are friendliness, gregariousness, assertiveness, activity level, excitement-seeking and cheerfulness. Extrovert people (high score on the E factor) tend to react with enthusiasm and often have positive emotions while introverted people (low score on the $\mathrm{E}$ factor) tend to be quiet, low-key and disengaged in social interactions.

Agreeableness (A) reflects individual differences in concern with cooperation and social harmony. The sub-domains of the A factor are trust, morality, altruism, cooperation, modesty and sympathy.

Neuroticism (N) refers to the tendency of experiencing negative feelings. People with high $\mathrm{N}$ values are emotionally reactive. They tend to respond emotionally to relatively neutral stimuli. They are often in a bad mood, which strongly affects their thinking and decision making (see Ch. ?? for more on decision making). Low $\mathrm{N}$ scorers are calm, emotionally stable and free from persistent bad mood. The subfactors are anxiety, anger, depression, self-consciousness, immoderation and vulnerability. The neuroticism factor is sometimes referred to as emotional stability [25].

The five factors and their respective adjectives are shown in Tab. 1.

\subsection{Other models of personality}

Other personality models that can be of interest to the recommender system community are the vocational RIASEC (with the main types Realistic, Investigative, Artistic, Social, Enterprising and Conventional) model [27], which was used in an e-commerce prototype [7] and the Bartle model (with the main types Killers, Achievers, Explorers and Socializers), which is suitable for the videogames domain [65].

The Thomas-Kilman conflict mode personality model has been developed to model group dynamics [66]. The model is composed of the following two dimensions that account for differences in individual behaviour in conflict situations ${ }^{1}$ :

1 The Thomas-Kilman conflict mode instrument is available at http: //cmpresolutions.co.uk/wp-content/uploads/2011/04/

Thomas-Kilman-conflict-instrument-questionaire.pdf 
Assertivness and Cooperativeness. Within this two-dimensional space subjects are classified into any of these five categories: Competing, Collaborating, Compromising, Avoiding or Accomodating.

Although learning styles per se are not considered as a personality model they share with personality the quality of being time invariant. In the domain of elearning (see also Ch. ??), models of learning styles have been used to recommend course material to students [17]. An example is the Felder and Silverman Learning Style Model [20] which measures four factors: active/ reflective, sensing/intuitive, visual/verbal and sequential/global.

In addition, some ad-hoc personality models have been proposed in the recommender systems community. For a trendy pictures recommender system, a personality model with two types, the trend-setters and the trend-spotters, has been proposed, along with a methodology for predicting the personality types from social media networks [62]. Especially in the domain of social networks, there is a tendency to stress the influence/susceptibility aspects of users as the main personality traits (e.g. leaders/followers) [5].

\begin{tabular}{|c|c|c|c|}
\hline Author & Model Name & $\begin{array}{l}\text { Primary } \\
\text { main }\end{array}$ & Main Types/Traits \\
\hline$\overline{[44]}$ & Five Factor Model & General & $\begin{array}{l}\text { Openness, Conscientiousness, Extraversion, } \\
\text { Agreeableness, and Neuroticism }\end{array}$ \\
\hline [34] & Four Temperaments & General & $\begin{array}{l}\text { Choleric, Sanguinic, Melancholic and Phleg- } \\
\text { matic }\end{array}$ \\
\hline [27] & RIASEC & Vocational & $\begin{array}{l}\text { Realistic, Investigative, Artistic, Social, Enter- } \\
\text { prising and Conventional }\end{array}$ \\
\hline [65] & Bartle types & Video games & Killers, Achievers, Explorers and Socializers \\
\hline [20] & $\begin{array}{l}\text { Felder and Silverman } \\
\text { Learning Style Model }\end{array}$ & $\begin{array}{l}\text { Learning } \\
\text { styles }\end{array}$ & $\begin{array}{l}\text { active/reflective, sensing/intuitive, } \\
\text { sual/verbal, sequential/global }\end{array}$ \\
\hline [66] & $\begin{array}{l}\text { Thomas-Kilmann con- } \\
\text { flict model }\end{array}$ & $\begin{array}{l}\text { group/conflict } \\
\text { modeling }\end{array}$ & Assertiveness, Cooperativeness \\
\hline
\end{tabular}

Table 2: Main personality models

\subsection{How does personality relate to user preferences?}

A number of studies showed that personality relates strongly with user preferences. Users with different personalities tend to prefer different kinds of content. These relations are domain dependant. Such an information is very valuable when designing a recommender system for a specific domain.

In their study, Rentfrow and Gosling [58] explored how music preferences are related to personality in terms of the FFM model. They categorized music pieces each into one of the four categories: reflective \& complex, intense \& rebelious, upbeat $\&$ conventional and energetic \& rhythmic. The reflective \& complex category was 
related to openness to new experience. Similarly, the intense \& rebelious category was also positively related to openness to new experience. However, although this category contains music with negative emotions it was not related to neuroticism or agreeableness. The upbeat \& conventional category was found to be positively related with extraversion, agreeableness, and Conscientiousness. Finally, they found that the energetic \& rhythmic category is related to extraversion and agreeableness.

In a similar study, Rentfrow et al. [57], extended the domain to general entertainment, which included music, books, magazines, films and TV shows. They categorized the content into the following categories: aesthetic, cerebral, communal, dark and thrilling. The communal category was positively related to extraversion, agreeableness and conscientiousness while being negatively related to extraversion and neuroticism. The aesthetic category was positively related to agreeableness, extraversion and negatively to neuroticism. The dark category was positively related extraversion and negatively to conscientiousness and agreeableness. The cerebral category was related to extraversion while the thrilling category did not reveal any consistent correlation with personality factors.

The relation between music and personality was also explored by Rawlings et al. [55]. They observed that the Extraversion and Openness factors are the only ones that explain the variance in the music preferences. Subjects with high openness tend to prefer diverse music styles. Extraversion, on the other hand, was found to be strongly related to preferences to popular music.

Cantador et al. [9] presented the results of an experiment where they observed the relations between user preferences and personality in the domains of movies, TV shows, music and books. Their work is based on the myPersonality dataset [37]. They observe a large number of relations between personality traits and individual domains as well as in crossed domains.

In an experiment based on a contextual movie recommender system dataset (the CoMoDa dataset [50]), Odić et al. explored the relations between personality factors and the induced emotions in movies in different social context [51]. They observed different patterns in experienced emotions for users in different social contexts (i.e. alone vs. not alone) as functions of the extraversion, agreeableness and neuroticism factors. People with different values of the conscientiousness and openness factors did not exhibit different patterns in their induced emotions.

\section{Personality acquisition}

The acquisition of personality parameters is the first major issue in the design of personality-based recommender systems. Generally, the acquisition techniques can be grouped into

- explicit techniques (questionnaires depending on the model)

- implicit techniques (regression/classification based on social media streams) 
While explicit techniques provide accurate assessments of the users' personalities they are intrusive and time consuming. Hence, these techniques are useful only in laboratory studies and for the assessment of ground truth data for the later automatic extraction.

Implicit techniques, on the other hand, offer an unobtrusive way of acquiring personality parameters. However, the accuracy of these instruments is not high and depends heavily on the quality of the source information (e.g. how often does a user tweet).

In this section we survey existing techniques for the acquisition of personality in recommender system. Tab. 3 sums the methods described in this section.

\begin{tabular}{|c|c|c|c|}
\hline Author & Method & Personality model & Source \\
\hline \multicolumn{2}{|c|}{$\begin{array}{l}{[23,26,33,15, \text { explicit }} \\
24,25]\end{array}$} & FFM & $\begin{array}{l}\text { Questionnaires (from } 10 \text { ques- } \\
\text { tions up) }\end{array}$ \\
\hline [53] & implict & FFM & micro-blogs (twitter) \\
\hline$[37,4,61]$ & implicit & FFM & social media (facebook) \\
\hline [21] & implicit & FFM & social media (weibo) \\
\hline [40] & implicit & FFM & role-playing game \\
\hline [16] & implicit & FFM & game (Commons Fishing Game) \\
\hline [11] & implicit & FFM & mobile phone logs \\
\hline [63] & implicit & FFM & emails \\
\hline [30] & implicit & FFM & ratings of products in a webstore \\
\hline [14] & implicit & FFM & stories \\
\hline [66] & explicit & $\begin{array}{l}\text { Thomas-Kilmann } \\
\text { flict model }\end{array}$ & con- questionnaire \\
\hline [64] & explicit & $\begin{array}{l}\text { Felder and Silve } \\
\text { Learning Style Mo }\end{array}$ & $\begin{array}{l}\text { rman questionnaire } \\
\text { lel }\end{array}$ \\
\hline
\end{tabular}

Table 3: Personality acquisition methods

\subsection{Explicit Personality Acquisition}

A widely used questionnaire for assessing the FFM factors is the International Personality Item Pool (IPIP) set of questionnaires [23]. The IPIP's web page ${ }^{2}$ contains questionnaires with 50 and 100 items, depending on the number of questions per factor (10 or 20). The relatively high number of questions makes it an accurate instrument, although it's time consuming for end users. Furthermore, it has been translated in many languages and validated in terms of cross-cultural differences [42].

In the questionnaire defined by Hellriegel and Slocum [26], each factor is measured via 5 questions, so there are 25 questions in total regarding the five factors.

2 http://ipip.ori.org/ 
Each factor's value is the average of user's scores on its related five questions. For example, the questions used to assess "Openness to Experience" include "imagination", "artistic interests", "liberalism", "adventurousness", and "intellect". Users are required to respond to every question on a 5-point Likert scale (for example, "imagination" is rated from 1 "no-nonsense" to 5 "a dreamer"). John and Srivastava [33] developed a more comprehensive list containing 44 items, called Big Five Inventory (BFI), by which each personality factor is measured by eight or nine questions. For example, the items related to "Openness to Experience" are "is original, comes up with new ideas", "is curious about many different things", "is ingenious, a deep thinker", "has an active imagination", etc. (each is rated on a 5-point Likert scale from "strongly disagree" to "strongly agree", under the general question of "I see Myself as Someone Who ..."). This questionnaire has been recognized as a wellestablished measurement of personality traits. The other commonly used public-free instruments include the 100-item Big Five Aspect Scales (BFAS) [15] and the 100 trait-descriptive adjectives [24]). A super-short measure of the Big5 model is the Ten Item Personality Inventory (TIPI) in which each factor is only assessed by two questions (e.g., "Openness to Experiences" is assessed by "open to new experiences, complex" and "conventional, uncreative" on the same Likert scale used in BFI) [25]. This instrument can meet the need for a very short measure (e.g., when time is limited), although it may somewhat diminished psychometric properties. We provide the TIPI questionnaire in Tab. 4.

\begin{tabular}{ll}
\hline FFM factor & Statement: I see myself as \\
\hline E & Extraverted, enthusiastic. \\
A & Critical, quarrelsome. \\
C & Dependable, self-disciplined. \\
N & Anxious, easily upset. \\
O & Open to new experiences, complex. \\
E & Reserved, quiet. \\
A & Sympathetic, warm. \\
C & Disorganized, careless. \\
N & Calm, emotionally stable. \\
O & Conventional, uncreative. \\
\hline
\end{tabular}

Table 4: The Ten-Items Personality Inventory Questionnaire developed by Gosling et al. [25].

A typical example of a commercially controlled instrument is the NEO PI-R (with a 240-items inventory) [12], which can not only measure the five factors, but also the six facets (i.e. subfactors) of each factor. For example, "Extroversion" contains six facets: Gregariousness (sociable), Assertiveness (forceful), Activity (energetic), Excitement-seeking (adventurous), Positive emotions (enthusiastic), and Warmth (outgoing). The NEO-FFI instrument, which measures the five factors only (but not their related facets), is a 60-item truncated version of NEO PI-R [12]. 
A quasi-explicit instrument for measuring personality is the approach of using stories. In their work, Dennis et al. [14] developed a set of stereotypical stories where each one conveys a personality trait from the FFM. For each of the five FFM factors they devised a pair of stories, one for a high level of the observed factor and one for the low level of the observed factor. The subject then rates how well each story applies to her/him on a Likert scale from 1 (extremely inaccurate) to 9 (extremely accurate).

Though different instruments have been developed so far, the choice of instrument is highly application-dependent and there is no one-size-fits-all measure.

\subsection{Implicit Personality Acquisition}

In their work, Quercia et al. [53], present the outcomes of a study that shows strong correlations between features extracted from users' micro-blogs and their respective FFM factors. The authors used the myPersonality dataset of 335 users. The dataset contains the users' FFM personality factors and the respective micro-blogs. The authors extracted several features from the micro-blogs and categorized them into the following quantities: listeners, popular, highly-read and influential. Each of these quantities showed a strong correlation with at least one of the FFM factors. The authors went a step further into predicting the FFM factors. Using a machine learning approach (the M5 rules regression and the 10 fold-cross validation scheme) they were able to achieve a predictability in RMSE ranging from 0.69 to 0.88 (on FFM factors ranging from 1 to 5).

Kosinski et al. [37] used the whole myPersonality dataset of over 58,000 subjects with their respective Facebook activity records to predict the FFM factors of the subjects. The source dataset was the user-like matrix of Facebook likes. The authors applied the Singular Value Decomposition method to reduce the number of features and used the logistic regression model to predict the FFM factors (along with other user parameters such as gender, age etc.). Their model was able to predict well the traits Openness and Extraversion while the other traits were predicted with lower accuracy.

An interesting approach was taken by van Lankveld et al. [40] who observed the correlation between FFM parameters and the users' behaviour in a videogame. They modified the Neverwinter Nights (a third-person role-playing video game) in order to store 275 game variables for 44 participants. They used variables that recorded conversation behavior, movement behavior and miscellaneous behaviors. They found significant correlations between all five personality traits and game variables in all groups.

Chittaranjan et al. [11] used mobile phone usage information for inferring FFM parameters. They used call logs (e.g. outgoing calls, incoming calls, average call duration etc.), SMS logs and application-usage logs as features for predicting the FFM factors. They observed that a number of these features have a significant correlations with the FFM factors. Using the Support Vector Machine classifier they achieved 
better results in the prediction of the traits than a random baseline although the difference was not always significant, which makes the task of inferring personality from call logs a hard one.

Shen et al. [63] attempted to infer the email writer's personality from her/his emails. To preserve privacy, they only extract high-level aggregated features from email contents, such as bag-of-word features (built from most commonly used words in daily life), meta features (such as TO/CC/BCC counts, importance of the email, count of different punctuation symbols, count of words, count of positive and negative numbers, count of attachments, month of the sent time, etc.), word statistics (through part-of speech tagging, sentiment analysis, and counting of pronouns and negations words), writing styles (in greeting patterns, closing patterns, wish patterns, and smiley words), and speech act scores (for detecting the purpose of workrelated emails). These groups of features are then applied to train predictors of the writer's personality, through three different generative models: joint model, sequential model, and survival model. The function is formally represented as $f: X \rightarrow Y$, where $X$ is the feature vector and $Y=\left\langle y_{1}, . ., y_{K}\right\rangle$ is the personality trait value vector (each element of $Y$ corresponds to one personality trait, such as Extraversion, with either of three values "low", "medium" and "high"). The joint model takes all the personality traits as a single entity to jointly decide whether a feature is selected; sequential model first selects a personality trait, and then uses this trait to decide whether to select a feature; survival model allows all personality traits to decide whether to select a feature independently, then the feature selected by all traits will be get selected. The experiment done on over 100,000 emails showed that the survival model (with label-independence assumption) works best in terms of prediction accuracy and computation efficiency, while joint model performs worst in terms of inferring personality traits such as Agreeableness, Conscientiousness, and Extraversion. The results to some extend infer that the personality traits are relatively distinct and independent from each other. Furthermore, it was found that people with high Conscientiousness are inclined to write long emails and use more characters; people with high Agreeableness tend to use more "please" and good wishes in their emails; and people with high Neuroticism use more negations.

The set of studies by Oberlander et al. [47,32] showed that personality can be inferred also from blog entries. In [32] they used features such as stemmed bigrams, no exclusion of stopwords (i.e. common words) or the boolean presence or absence of features noted (rather than their rate of use) in combination with the Support Vector Machines classifier. On a large corpus of blogs they managed to predict the FFM factors with an accuracy ranging from $70 \%$ (for neuroticism) to $84 \%$ (for openness).

With the development of social networking, some researchers have begun to study the correlation between users' personality and their social behavior on the web (e.g., Facebook, Twitter) [4, 59]. For example, [4] found strong connection between users' personality and their Facebook use through a user survey on 237 students. Participants' personality was self-reported through answering the NEO PI-R questionnaire. The collected personality data were then used to compute correlation with users' Facebook information (such as basic information, personal information, contact information and education, and work information). The results show that 
Extroversion has a positive effect on the number of friends. Moreover, individuals with high Neuroticism are more inclined to post their private information (such as photos). The factor Openness to Experience was found to have positive correlation with users' willingness to use Facebook as a communication tool, and the factor Conscientiousness is positively correlated with the number of friends. In [61], a similar experiment was performed. They verified again that Extroversion was significantly correlated with the size of a user's social network. Moreover, people tend to choose friends who are with higher Agreeableness but similar Extroversion and Openness.

In [22], the authors developed a method to predict users' personality from their Facebook profile. Among various features, they identified ones that have a significant correlation with one or more of the Big5 personality traits based on studying 167 subjects' public data on Facebook. These features include linguistic features (such as swear words, social processes, affective processes, perceptual processes, etc.), structural features (number of friends, egocentric network density), activities and preferences (e.g., favorite books), and personal information (relationship status, last name length in characters). Particularly, the linguistic analysis of profile text (which is the combination of status updates, About Me, and blurb text) was conducted through Linguistic Inquiry and Word Count (LIWC) program [52], which is a tool to produce statistics on 81 different text features in five psychological categories. They further proposed a regression analysis based approach to predict the personality, in two variations: M5'Rules, and Gaussian Processes. The testing shows that the prediction of each personality factor can be within $11 \%$ of the actual values. Moreover, M5'Rules acts more effective than Gaussian Processes, with stronger connection to Openness, Conscientiousness, Extroversion, and Neuroticism.

Recently, Gao et al. [21] proposed a method for inferring the users' personality from their social media contents. To be specific, they obtained 1766 volunteers' personality values and Weibo behavior (which is a popular micro-blog site in China) to train the prediction model. 168 features were extracted from these users' Weibo status, and then classified into categories including status statistics features (e.g., the total number of statuses), sentence-based features (the average number of Chinese characters per sentence), word-based features (the number of emotion words), character-based features (the number of commas, colons, etc.), and LIWC features. They then applied M5-Rules, Pace Regression and Gaussian process, to make prediction. The results show that the Pearson correlation between predicted personality and user self-reported personality can achieve 0.4 (i.e., fairly correlated), especially regarding the three traits Conscientiousness, Extroversion, and Openness to Experience.

$\mathrm{Hu}$ and $\mathrm{Pu}$ studied the effect of personality on users' rating behavior in recommender systems [30]. They obtained 86 participants' valid ratings on at least 30 items among a set of 871 products (from 44 primary categories). The rating behavior was analyzed from four aspects: number of rated items (NRI), percentage of positive ratings (PerPR), category coverage (CatCoverage), and interest diversity (IntDiversity). The CatCoverage is measured as the number of categories of rated items. The IntDiversity reveals the distribution of users' interests in each category, formally 
defined as the Shannon index according to information theory. They calculated the correlation between users' Big5 personality traits and the rating variables through Pearson product-moment. The results identify the significant impact of personality on the way users rate items. Particularly, Conscientiousness and gender were found negatively correlated with the number of ratings, category coverage and interest diversity, which indicates that conscientious and/or female users are more likely to prefer providing fewer ratings, lower level of category coverage, and lower interest diversity. In addition, Agreeableness is positively correlated with the percentage of positive ratings, implying that agreeable people tend to give more positive ratings. All these findings show correlations between personality and rating behaviour on the samples used. However, exploring whether it is possible to infer personality from rating behaviour is an open issue for future work.

Dunn et al. [16] proposed, beside an explicit questionnaire, a gamified user interface for the acquisition of personality for recommender systems. Through the Commons Fishing Game (CFG) interface the users were instructed to maximize the amount gathered from a common resource, which was shared amongst a group of players; collectively trying not to deplete this resource. The experiment showed that it is possible to predict Extraversion and Agreeableness with the described instrument.

\subsection{Datasets for offline recommender systems experiments}

Given that a number of research activities has already been published, there exist some datasets that can be used for personality-aware recommender systems experiments. The minimal requirements for such a dataset are (a) to include the user-item interaction data (e.g. ratings) and (b) to include the personality factors associated to the users. In this subsection we survey a number of such datasets, which are summarized in Tab. 5.

The first dataset containing personality parameters to be released was the LDOSPerAff-1 [71]. Based on 52 subjects it contains ratings of images. The user-item matrix has all elements (i.e. sparsity is null). The dataset contains the corresponding FFM factors for each user. The FFM factors were acquired using the 50-items IPIP questionnaire [23]. Furthermore, all items were selected from the IAPS dataset of images [39] and are annotated with the values of the induced emotions in the valence-arousal-dominance (VAD) space.

The LDOS-CoMoDa (Context Movies Dataset) dataset [38] was developed for research on contextual recommender systems. A unique feature of the dataset is that it contains FFM parameters for each users. According to [38] it contains data for 95 users and 961 movies. The FFM factors were collected using the 50-items IPIP questionnaire [23]. The dataset is also rich in contextual parameters such as time, weather, location, emotions, social state etc.

A dataset that contains more users is the myPersonality dataset [37]. It contains FFM factors for 38,330 users. The dataset has been collected using a Facebook 
application. It contains the Facebook Likes for each of the users. Furthermore it also contains twitter names for more than 300 subjects which opens new possibilities for crawling these users' micro-blogs (as has been done in [53]).

Chittaranjan et al. [11] presented a dataset of mobile phone users logs along with the respective FFM values. The dataset contains information about 177 subjects and their daily phone usage activities (the CDR - call data record) over a period of 17 months on a Nokia N95 smartphone. The phone usage logs contain data related to calls, SMSs and application usage.

Furthermore, a number of datasets, not released as datasets per se, exist, as they have been used in the studies reported in this chapter.

\begin{tabular}{|c|c|c|c|c|c|}
\hline Name & Author & Domain & $\begin{array}{l}\text { Personality } \\
\text { model }\end{array}$ & $\begin{array}{l}\text { Number } \\
\text { of } \\
\text { subjects }\end{array}$ & Other metadata \\
\hline LDOS-CoMoDa & [38] & Movies & FFM & 95 & $\begin{array}{l}\text { movie context metadata } \\
\text { (location, weather, social } \\
\text { state, emotions etc.) }\end{array}$ \\
\hline LDOS-PerAff-1 & [71] & Images & FFM & 52 & $\begin{array}{l}\text { item induced emotions in } \\
\text { the VAD space }\end{array}$ \\
\hline myPersonality & [37] & $\begin{array}{l}\text { Social Media } \\
\text { (Facebook) }\end{array}$ & FFM & 38330 & twitter names \\
\hline Chittaranjan & [11] & $\begin{array}{l}\text { Mobile phone } \\
\text { usage }\end{array}$ & FFM & 117 & $\begin{array}{l}\text { call logs, SMS logs, app } \\
\operatorname{logs}\end{array}$ \\
\hline
\end{tabular}

Table 5: Overview of datasets

\section{How to use personality in recommender systems}

In this section we provide an overview of how personality has been used in recommender systems. The most common issues addressed are the cold-start problem and the presentation of the recommended results in terms of diversity. Tab. 6 summarizes the various strategies described in this section.

\subsection{Addressing the new user problem}

The new user problem occurs when the recommender system does not have enough ratings from a user that has just started to use the system [3]. The problem is present both in content-based recommender systems and in collaborative recommender systems although it is more difficult to solve within the latter. The system must first have some information about the user, which is usually in the form of ratings. In the case of content-based recommender systems, the lack of ratings implies that, 


\begin{tabular}{|c|c|c|c|}
\hline Author & Recommender System's Goal & $\begin{array}{l}\text { Personality acquisi- } \\
\text { tion method }\end{array}$ & Approach \\
\hline [72] & cold-start problem & IPIP 50 & user-user similarity measure based on personality \\
\hline [29] & cold-start problem & TIPI & user-user similarity measure based on personality \\
\hline$[18,8]$ & cold-start problem & TIPI & active learning, matrix factorization \\
\hline [74] & diversity & TIPI & $\begin{array}{l}\text { personality-based diversity adjusting approach for } \\
\text { movie recommendation }\end{array}$ \\
\hline [67] & diversity & NEO IPIP 20 & personality-based diversity adaptation \\
\hline [9] & cross-domain recommendations & NEO IPIP 20 & $\begin{array}{l}\text { similarities between personality-based user stereo- } \\
\text { types for genres in different domains }\end{array}$ \\
\hline$[56,54]$ & group recommendations & $\begin{array}{l}\text { Thomas-Kilmann } \\
\text { conflict model } \\
\text { instrument }\end{array}$ & $\begin{array}{l}\text { combining assertiveness and cooperativeness into } \\
\text { the aggregation function }\end{array}$ \\
\hline [35] & group recommendations & $\begin{array}{l}\text { Thomas-Kilmann } \\
\text { conflict model in- } \\
\text { strument and NEO } \\
\text { IPIP } 20\end{array}$ & $\begin{array}{l}\text { group satisfaction modeling with a personality- } \\
\text { based graph model }\end{array}$ \\
\hline
\end{tabular}

Table 6: Survey of recommender systems using personality

for the observed user, the system does not know the preferences towards the item's features (e.g. the genre). In the case of collaborative filtering, especially in neighborhood methods, the lack of ratings for a new user implies that there are not enough overlapping ratings with other users, which makes it hard to calculate user similarities. So far this problem has been tackled with various techniques such as hybrid methods [3], adaptive learning techniques [19] or simply by recommending popular items [3].

Personality is suitable to address the new-user problem. Given the assumption that the user's personality is available (e.g. from another domain) it can be used in collaborative filtering recommender systems.

Personality has been used in a memory-based collaborative filtering recommender system for images [72, 69]. In an offline experiment, the authors acquired explicit FFM parameters for each user and calculated the user distances (as opposed to similarities) using the weighted distance formula

$$
d\left(b_{i}, b_{j}\right)=\sqrt{\sum_{l=1}^{5} w_{l}\left(b_{i l}-b_{j l}\right)^{2}}
$$

where $b_{i}$ and $b_{j}$ are the FFM vectors for two arbitrary users $\left(b_{i l}\right.$ and $b_{j l}$ are the individual FFM factors) and $w_{l}$ are the weights. The weights were computed as the eigenvalues from the principal component analysis on the FFM values of all users. On the given dataset, this approach was statistically equivalent to using standard rating-based user similarity measures.

A similar approach was taken by $\mathrm{Hu}$ and $\mathrm{Pu}$ [29] where they used a different formula to calculate the user similarities. They proposed to use the Pearson correlation coefficient to calculate the user similarities

$$
\operatorname{sim}\left(b_{i}, b_{j}\right)=\frac{\sum_{l}\left(b_{i l}-\overline{b_{i}}\right)\left(b_{j l}-\overline{b_{j}}\right)}{\sqrt{\sum_{l}}\left(b_{i l}-\overline{b_{i}}\right)^{2} \sqrt{\sum_{l}}\left(b_{j l}-\overline{b_{j}}\right)^{2}}
$$


and they combined it with existing ratings by controlling the contribution of each similarity measure with the weight $\alpha$. They compared the proposed approach to a rating-based user similarity metric collaborative filtering recommender system. On a dataset of 113 users and 646 songs the personality-based algorithm outperformed the rating-based in terms of mean absolute error, recall and specificity.

Our results showed that both personality-based similarity and the hybrid scheme lead CF recommender systems to generate more accurate recommendations than the traditional rating-based one in a sparse music dataset. In

A standard approach to tackle the cold-start problem is to use the active learning approach (rating elicitation - see also Ch. ??) [19]. In their work, Elahi et al. [18], proposed an active learning strategy that incorporated user personality data. They acquired the personality information using the 10-items IPIP questionnaire through a mobile application. They formulated the rating prediction as a modified matrix factorization approach where the FFM factors are treated as additional users' latent factors:

$$
\hat{r}_{u i}=b_{i}+b_{u}+q_{i}^{T} \cdot\left(p_{u}+\sum_{l} b_{l}\right)
$$

where $p_{u}$ is the latent factor of the user $u, q_{i}$ is the latent factor o the item $i, b_{u}$ and $b_{i}$ are the user's and item's biases and $b_{l}$ are the FFM factors. The proposed rating elicitation method outperformed (in terms of Mean Absolute Error) the baseline (the $\log$ (popularity)*entropy method) and the random method.

In these examples, personality has been acquired separately with questionnaires. With this approach the authors have just moved the burden of an initial questionnaire about user ratings to another initial questionnaire (for personality). However, the idea here is that personality is going to be available in advance, for example from other domains or acquired implicitly.

\subsection{Diversity/serendipity}

Recently, the impact of personality on users' preferences on recommendation diversity has been investigated in [10,67]. Diversity refers to recommending users a diverse set of items, so as to allow them to discover unexpected items more effectively [46] (see also Ch. ??). The existing approaches commonly adopt a fixed strategy to adjust the diversity degree within the set of recommendations [75, 31, 2], which however, does not consider that different users might possess different attitudes towards the diversity of items. The limitation motivates the authors of paper [10] to research whether and how personality might impact users' needs for diversity in recommender systems. They conducted a user survey (with 181 subjects) to know the causal relationship. For each user, they obtained her/his movie selections as well as personality values. Then, two levels of diversity were considered: the diversity in respect to individual attributes (such as the movie's genre, director, actor/actress, etc.); the overall diversity when all attributes are combined. The correlation analy- 
sis showed that some personality factors have a significant correlation with users' diversity preferences. For instance, it shows that more reactive, excited and nervous persons (high Neuroticism) are more inclined to choose diverse directors, and suspicious/ antagonistic users (low Agreeableness) prefer diverse movie countries. As for the movie's release time, its diversity is preferred by efficient/organized users (high Conscientiousness), while for the movie's actor/actress, its diversity is preferred by imaginative/creative users (high Openness to experience). At the second level (i.e., overall diversity), no matter how the weights placed on attributes vary, Conscientiousness was shown significantly negatively correlated with it, which means that less conscientious people essentially prefer higher level of overall diversity.

Inspired by the user survey's findings, they developed a personality-based diversity adjusting approach for movie recommendation [74]. They have incorporated personality, as a moderating factor, into a content-based recommender system. Specifically, given the user's personality values in respect to the Big5 factors, they first identify her/his diversity needs. For example, since high Openness to Experience is linked to high need for diversity with regards to "actor/actress", in the case that the "actor/actress" is the current user's most important attribute and s/he possesses a high Openness to Experience value, the system will return movies with diverse actors/actresses to the user. In addition, if the user has a low Conscientiousness value, the system will further increase the recommendations' overall diversity degree, since low Conscientiousness is correlated with high need for the overall diversity. The number of diverse items within the whole recommendation set is accordingly adjusted to reflect the user's diversity needs. The proposed method was tested in a controlled user study (with 52 participants), by means of comparing it to a variant that incorporated personality in the contrary way (i.e., offering less diverse items to the user though s/he spontaneously requires a higher level of diversity given her/his personality values). The user evaluation demonstrated that their method can significantly increase users' perception of system competence and recommendation accuracy. Users were also more satisfied with the personality-based recommendation. The findings thus consolidate the previous survey's results. They also suggest an effective solution in terms of taking personality into account for generating personalized diversity in recommender systems.

Tintarev et al. applied a User-as-Wizard approach to study how people apply diversity to the set of recommendations [67]. Particularly, they emphasized the personality factor Openness to Experience as for its specific role in personalizing the recommendation diversity's level, because it describes users' imagination, aesthetic sensitivity, attentiveness to inner feelings, preference for variety, and intellectual curiosity (so they assumed that people with higher Openness to Experience would be more willing to receive novel items). Their experiment was in the form of an online questionnaire with the aid of Amazon's Mechanical Turk (MT) service. 120 users' responses were analyzed. Each of them was required to provide some recommendation to a fictitious friend who is in one of three conditions: high Openness to Experience, low Openness to Experience, no personality description (baseline). The results did not prove the effect of Openness to Experience on the overall diversity participants applied, but the authors observed that participants tend to recommend 
items with high categorical diversity (i.e., across genres) but low thematic diversity (inter-genre) to others who are more open to experience. In other words, users who are low on Openness to Experience might prefer thematic diversity to categorical variation. The observation is consistent with the finding from [1] that users generally prefer recommendations from diversified categories, but less diversity within one category.

\subsection{Cross-domain recommendations}

As we mentioned in the introduction, personality is domain-independent, i.e. when users are being recommended books or movies, we can use the same personality profile. This can be especially useful in cross-domain recommender systems (see also Ch. ??). In a study performed by Cantador et al. [9] personality factors are related to domain genres and similarities between personality-based user stereotypes for genres in different domains are computed. Among the many cross-domaingenres combinations we can find relations such as salsa-music lovers are dissimilar to science-fiction-books lovers or news-tv-show lovers are similar to mystery-books lovers.

\subsection{Group Recommender Systems}

Group recommendations are discussed in Ch. ??. Recommending items to groups of users is not the same as recommending items to individual users [41]. Beside having to choose among strategies that address users as individuals (e.g. least misery, most pleasure etc. - see Ch. ?? for an extensive overview), the relationships between group members play an important role. Personality is an important factor in group dynamics.

In their work, Garcia, Sanchez et al.. [56, 54] propose to use the ThomasKilmann Conflict personality model [66] to model the relationships between group members in terms of assertiveness and cooperativeness. They applied the model to three group recommendation approaches (i.e. least misery, minimize penalization and average satisfaction). They collected ground truth data through a user study with 70 students who formed groups, discussed and decided which movies they would watch together in a cinema. The proposed approach showed an increase in prediction accuracy compared to the same techniques without taking into account the conflict personality model.

Similarly, Kompan et al. [35] used the Thomas-Kilmann model and the FFM to model individual users. They modeled the group satisfaction with a graph-based approach where vertices represent users and edges represent user influences based on relationship, personality and actual context. They performed a small-scale user study with users rating movies. The usage of the personality-based group satisfac- 
tion model in an average-aggregation strategy-based group recommender system outperformed the same algorithm without the proposed group satisfaction modeling.

\section{Open issues and Challenges}

The usage of personality in recommender systems has just started, which makes it a very interesting research topic as there are quite some open issues and challenges that need to be addressed. In this section we survey these open issues.

\subsection{Non-intrusive acquisition of personality information}

The limitation of traditional explicit acquisition approach is that the required user effort is usually high, especially if we want to obtain their accurate personality profile (e.g., through 100-item Big Five Aspect Scales (BFAS); see Section 3.1). Users might be reluctant to follow the time-consuming and tedious procedure to answer all questions, due to their cognitive or emotional reason. Thus, the implicit, unobtrusive approach might be more acceptable and effective to build their personality profile. The critical question is then how to accurately derive users' personality traits from the information they have provided. In Section 3.2, we discussed various methods, such as ones based on users' emails or their generated contents and behavior in social networking sites (e.g., Facebook, Twitter). However, the research is still at the beginning stage, and there is large room to improve the existing algorithms' accuracy. One possible solution is to explore other types of info as to their power of reflecting users' personality. For instance, since the significant correlation between users' personality and their rating behavior was proven in [30], the findings might be constructive for some researchers to develop the rating-based personality inference algorithm. The developed method might be further extended to consider the possible impacts of other actions, such as users' browsing, clicking, and selecting behavior in recommender systems. Indeed, it will be interesting to investigate the complementary roles of various resources to fulfill their combinative effect on deriving users' personality. To be specific, we may infer users' personality by integrating their history data left at different platforms (e.g., the integration of rating behavior, email, and social media content). The different types of info might be heterogenous in nature, so how to effectively fuse them together might be an open issue. 


\subsection{Larger datasets}

The recommender systems oriented datasets containing personality factors of users are very few (see Sec. 3.3). Furthermore the number of subjects in databases is very low, ranging from roughly 50 to a little more than 100, with the exception of the myPersonality dataset. Compared to the huge datasets that the recommender systems community is used to work with (e.g. the Netflix or the Yahoo! Music datasets) the lack of bigger datasets is an obvious issue that needs to be addressed.

\subsection{Cross-domain applications}

An unexplored area of recommender systems, where personality appears to be a natural fit, are cross-domain applications (see also Ch. ??). As personality is domainindependent it can be used as a generic user model. Cross-domain applications have been researched in the past and correlations of preferences among different domains have been identified. For example, Winoto et al. [73] observed the relations between the games, TV series and movie domains, while Tiroshi et al. [68] observed the relations between music, movies, TV series and books. The first to explore the potential role of personality in cross-domain applications were Cantador et al. [9] who observed the relations between the FFM factors and preferences in various domains (movies, TV shows, music, books). An intuitive continuation of this work is the application of the personalities learned in one domain to another domain to beat the cold-start problem.

Another aspect of cross-domain recommendations is cross-application recommendations. In order to be able to transfer the personality profiles between applications a standardized description of personality should be used. There has been an attempt, the Personality Markup Language (PersonalityML), to standardize the description of personality in user models across different domains [6].

\subsection{Diversity}

How to provide diverse and novel recommendations has increasingly become an important topic in the area of recommeder systems. That is, we are no longer satisfied with providing items similar to what users preferred before, but showing ones that can be unexpected and surprising to users. The recent works $[10,67]$ have indicated the difference occurring among users in terms of their needs for recommendation diversity as influenced by their inherent personality. It hence comes to the question of how to enhance the existing diversity algorithm to make it more tailored to individual user's requirement. For example, in [74], the authors gave a preliminary attempt to solve this problem and obtain interesting results. The ideas might be further enhanced and consolidated from both aspects of algorithm development and 
user evaluation. Moreover, in addition to personality, it will be meaningful to study the potential influence of other personal factors such as demographic characteristics (e.g., age, gender, cultural background). According to [10], some demographical properties did show significant correlation with some diversity variables. For example, people who are younger and/or with lower education level are more likely to prefer diverse movies. It hence suggests that these factors could be considered together with personality for optimally adjusting the diversity degree within the list of recommendations.

\subsection{Privacy issues}

Although all the research done so far on personality in recommender systems touched upon the sensitivity of the data, the issue of privacy has not been addressed properly yet. The fact that, in terms of personality, a user can be tagged as neurotic or otherwise with labels that suggest a negative trait makes these data very sensitive. Schrammel et al. [60] explored if there were any differences in the degree of disclosure acceptance among users with different personalities but found no significant differences. Some aspects are discussed in Ch. ??.

\section{Conclusion}

In this chapter we presented the usage of personality in recommender systems. Personality, as defined in psychology, accounts for the most important ways in which users differ in their preferences and behaviour. It can be acquired using either questionnaires or by inferring implicitly from other sources (e.g. social media streams). The most common model of personality is the Five Factor Model (FFM), which is composed of the factors openness, conscientiousness, extraversion, agreeableness and neuroticism. This model is suitable for recommender systems since it can be quantified with feature vectors that describe the degree each factor is expressed in a user. Furthermore, the FFM (and personality in general) is domain independent. We presented several methods for the acquisition of personality factors, with a special focus on implicit methods. We showcased a number of ways recommender systems have been shown to improve using personality models, especially in terms of the cold-start problem and diversity. Finally, we provided a list of open issues and challenges that need to be addressed in order to improve the adoption of personality in recommender systems.

Acknowledgements Part of the work presented in this chapter has received funding from the European Union FP7 programme through the PHENICX project (grant agreement no. 601166), China National Natural Science Foundation (no. 61272365), and Hong Kong Research Grants Council (no. ECS/HKBU211912). 


\section{References}

1. Abbassi, Z., Mirrokni, V.S., Thakur, M.: Diversity maximization under matroid constraints. In: Proceedings of the 19th ACM SIGKDD International Conference on Knowledge Discovery and Data Mining, KDD '13, pp. 32-40. ACM, New York, NY, USA (2013). DOI 10.1145/ 2487575.2487636

2. Adomavicius, G., Kwon, Y.: Improving aggregate recommendation diversity using rankingbased techniques. Knowledge and Data Engineering, IEEE Transactions on 24(5), 896-911 (2012). DOI 10.1109/TKDE.2011.15

3. Adomavicius, G., Tuzhilin, a.: Toward the next generation of recommender systems: a survey of the state-of-the-art and possible extensions. IEEE Transactions on Knowledge and Data Engineering 17(6), 734-749 (2005). DOI 10.1109/TKDE.2005.99

4. Amichai-Hamburger, Y., Vinitzky, G.: Social network use and personality. Computers in $\mathrm{Hu}-$ man Behavior 26(6), 1289-1295 (2010)

5. Aral, S., Walker, D.: Identifying influential and susceptible members of social networks. Science (New York, N.Y.) 337(6092), 337-41 (2012). DOI 10.1126/science.1215842

6. Augusta Silveira Netto Nunes, M., Santos Bezerra, J., Adicinéia, A.: PersonalityML: A Markup Language to Standardize the User Personality in Recommender Systems. Revista Gestão, Inovação e Tecnologia 2(3), 255-273 (2012). DOI 10.7198/ S2237-0722201200030006

7. Bologna, C., Rosa, A.C.D., Vivo, A.D., Gaeta, M., Sansonetti, G., Viserta, V., A, Q.G.S.: Personality-Based Recommendation in E-Commerce. EMPIRE 2013: Emotions and Personality in Personalized Services (2013)

8. Braunhofer, M., Elahi, M., Ge, M., Ricci, F.: Context Dependent Preference Acquisition with Personality-Based Active Learning in Mobile Recommender Systems. Learning and Collaboration Technologies. Technology-Rich Environments for Learning and Collaboration pp. 105-116 (2014). DOI 10.1007/978-3-319-07485-6\_11

9. Cantador, I., Fernández-tobías, I., Bellogín, A.: Relating Personality Types with User Preferences in Multiple Entertainment Domains. EMPIRE 1st Workshop on "Emotions and Personality in Personalized Services", 10. June 2013, Rome (2013)

10. Chen, L., Wu, W., He, L.: How personality influences users' needs for recommendation diversity? CHI ' 13 Extended Abstracts on Human Factors in Computing Systems on - CHI EA '13 p. 829 (2013). DOI 10.1145/2468356.2468505

11. Chittaranjan, G., Blom, J., Gatica-Perez, D.: Mining large-scale smartphone data for personality studies. Personal and Ubiquitous Computing 17(3), 433-450 (2011). DOI 10.1007/s00779-011-0490-1

12. Costa, P.T., Mccrae, R.R.: NEO PI-R professional manual. Odessa, FL (1992)

13. Deniz, M.: An Investigation of Decision Making Styles and the Five-Factor Personality Traits with Respect to Attachment Styles. Educational Sciences: Theory and Practice 11(1), 105-114 (2011)

14. Dennis, M., Masthoff, J., Mellish, C.: The quest for validated personality trait stories. In: Proceedings of the 2012 ACM international conference on Intelligent User Interfaces - IUI '12, p. 273. ACM Press, New York, New York, USA (2012). DOI 10.1145/2166966.2167016

15. DeYoung, C.G., Quilty, L.C., Peterson, J.B.: Between facets and domains: 10 aspects of the Big Five. Journal of personality and social psychology 93(5), 880-896 (2007). DOI 10.1037/ 0022-3514.93.5.880

16. Dunn, G., Wiersema, J., Ham, J., Aroyo, L.: Evaluating interface variants on personality acquisition for recommender systems. User Modeling, Adaptation, and Personalization pp. 259-270 (2009). DOI 10.1007/978-3-642-02247-0 \-25

17. El-Bishouty, M.M., Chang, T.W., Graf, S., Chen, N.S.: Smart e-course recommender based on learning styles. Journal of Computers in Education 1(1), 99-111 (2014). DOI 10.1007/ s40692-014-0003-0

18. Elahi, M., Braunhofer, M., Ricci, F., Tkalcic, M.: Personality-based active learning for collaborative filtering recommender systems. AI*IA 2013: Advances in Artificial Intelligence pp. 360-371 (2013). DOI 10.1007/978-3-319-03524-6\_31 
19. Elahi, M., Repsys, V., Ricci, F.: Rating elicitation strategies for collaborative filtering. ECommerce and Web Technologies pp. 160-171 (2011)

20. Felder, R., Silverman, L.: Learning and teaching styles in engineering education. Engineering education 78(June), 674-681 (1988)

21. Gao, R., Hao, B., Bai, S., Li, L., Li, A., Zhu, T.: Improving user profile with personality traits predicted from social media content. In: Proceedings of the 7th ACM Conference on Recommender Systems, RecSys '13, pp. 355-358. ACM, New York, NY, USA (2013). DOI $10.1145 / 2507157.2507219$

22. Golbeck, J., Robles, C., Turner, K.: Predicting personality with social media. Proceedings of the 2011 annual conference extended abstracts on Human factors in computing systems - CHI EA '11 p. 253 (2011). DOI 10.1145/1979742.1979614

23. Goldberg, L., Johnson, J., Eber, H., Hogan, R., Ashton, M., Cloninger, C., Gough, H.: The international personality item pool and the future of public-domain personality measures. Journal of Research in Personality 40(1), 84-96 (2006). DOI 10.1016/j.jrp.2005.08.007

24. Goldberg, L.R.: The Development of Markers for the Big-Five Factor Structure. Psychological assessment 4(1), 26-42 (1992)

25. Gosling, S.D., Rentfrow, P.J., Swann, W.B.: A very brief measure of the Big-Five personality domains. Journal of Research in Personality 37(6), 504-528 (2003). DOI 10.1016/ S0092-6566(03)00046-1

26. HellriegelDon, Slocum, J.: Organizational Behavior. Cengage Learning (2010)

27. Holland, J.L.: Making vocational choices: A theory of vocational personalities and work environments. Psychological Assessment Resources (1997)

28. Hu, R., Pu, P.: A Study on User Perception of Personality-Based Recommender Systems. User Modeling, Adaptation, and Personalization 6075, 291-302 (2010). DOI 10.1007/ 978-3-642-13470-8 \_27

29. Hu, R., Pu, P.: Using Personality Information in Collaborative Filtering for New Users. Recommender Systems and the Social Web p. 17 (2010)

30. $\mathrm{Hu}, \mathrm{R} ., \mathrm{Pu}, \mathrm{P} . \mathrm{E}$ Exploring Relations between Personality and User Rating Behaviors. EMPIRE 1st Workshop on "Emotions and Personality in Personalized Services", 10. June 2013, Rome (2013)

31. Hurley, N., Zhang, M.: Novelty and diversity in top-n recommendation - analysis and evaluation. ACM Trans. Internet Technol. 10(4), 14:1-14:30 (2011). DOI 10.1145/1944339. 1944341

32. Iacobelli, F., Gill, A.J., Nowson, S., Oberlander, J.: Large Scale Personality Classification of Bloggers. In: S. D'Mello, A. Graesser, B. Schuller, J.C. Martin (eds.) Affective Computing and Intelligent Interaction, Lecture Notes in Computer Science, vol. 6975, pp. 568-577. Springer Berlin Heidelberg, Berlin, Heidelberg (2011). DOI 10.1007/978-3-642-24571-8

33. John, O.P., Srivastava, S.: The Big Five trait taxonomy: History, measurement, and theoretical perspectives. In: L.A. Pervin, O.P. John (eds.) Handbook of personality: Theory and research, vol. 2, second edn., pp. 102-138. Guilford Press, New York (1999)

34. Keirsey, D.: Please Understand Me 2? Prometheus Nemesis pp. 1-350 (1998)

35. Kompan, M., Bieliková, M.: Social Structure and Personality Enhanced Group Recommendation. UMAP 2014 Extended Proceedings (2014)

36. Koren, Y., Bell, R., Volinsky, C.: Matrix Factorization Techniques for Recommender Systems. Computer 42(8), 30-37 (2009). DOI 10.1109/MC.2009.263

37. Kosinski, M., Stillwell, D., Graepel, T.: Private traits and attributes are predictable from digital records of human behavior. Proceedings of the National Academy of Sciences pp. 2-5 (2013). DOI 10.1073/pnas.1218772110

38. Košir, A., Odić, A., Kunaver, M., Tkalčič, M., Tasič, J.F.: Database for contextual personalization. Elektrotehniški vestnik 78(5), 270-274 (2011)

39. Lang, P.J., Bradley, M.M., Cuthbert, B.N.: International affective picture system (IAPS): Affective ratings of pictures and instruction manual. Technical Report A-8. Tech. rep., University of Florida (2005) 
40. van Lankveld, G., Spronck, P., van den Herik, J., Arntz, A.: Games as personality profiling tools. 2011 IEEE Conference on Computational Intelligence and Games (CIG'11) pp. 197202 (2011). DOI 10.1109/CIG.2011.6032007

41. Masthoff, J., Gatt, A.: In pursuit of satisfaction and the prevention of embarrassment: affective state in group recommender systems. User Modeling and User-Adapted Interaction: The Journal of Personalization Research 16(3-4), 281-319 (2006). DOI 10.1007/s11257-006-9008-3

42. McCrae, R., Allik, I.: The five-factor model of personality across cultures. Springer (2002)

43. McCrae, R.R., Costa, P.T.: A contemplated revision of the NEO Five-Factor Inventory. Personality and Individual Differences 36(3), 587-596 (2004). DOI 10.1016/S0191-8869(03) 00118-1

44. McCrae, R.R., John, O.P.: An Introduction to the Five-Factor Model and its Applications. Journal of Personality 60(2), p175 - 215 (1992)

45. McNee, S.M., Riedl, J., Konstan, J.A.: Being accurate is not enough. In: CHI '06 extended abstracts on Human factors in computing systems - CHI EA '06, p. 1097. ACM Press, New York, New York, USA (2006). DOI 10.1145/1125451.1125659

46. McNee, S.M., Riedl, J., Konstan, J.A.: Being accurate is not enough: How accuracy metrics have hurt recommender systems. In: CHI '06 Extended Abstracts on Human Factors in Computing Systems, CHI EA '06, pp. 1097-1101. ACM, New York, NY, USA (2006). DOI $10.1145 / 1125451.1125659$

47. Nowson, S., Oberlander, J.: Identifying more bloggers: Towards large scale personality classification of personal weblogs. International Conference on Weblogs and Social Media. (2007)

48. Nunes, M.A.S., Hu, R.: Personality-based recommender systems. In: Proceedings of the sixth ACM conference on Recommender systems - RecSys '12, p. 5. ACM Press, New York, New York, USA (2012). DOI 10.1145/2365952.2365957

49. Nunes, M.A.S.N.: Recommender Systems based on Personality Traits: Could human psychological aspects influence the computer decision-making process? VDM Verlag (2009)

50. Odić, A., Tkalčič, M., Tasic, J.F., Košir, A.: Predicting and Detecting the Relevant Contextual Information in a Movie-Recommender System. Interacting with Computers 25(1), 74-90 (2013). DOI 10.1093/iwc/iws003

51. Odić, A., Tkalčič, M., Tasič, J.F., Košir, A.: Personality and Social Context : Impact on Emotion Induction from Movies. UMAP 2013 Extended Proceedings (2013)

52. Pennebaker, J.W., Francis, M.E., Booth, R.J.: Linguistic inquiry and word count: Liwc 2001. Mahway: Lawrence Erlbaum Associates p. 71 (2001)

53. Quercia, D., Kosinski, M., Stillwell, D., Crowcroft, J.: Our Twitter Profiles, Our Selves: Predicting Personality with Twitter. In: 2011 IEEE Third Int'l Conference on Privacy, Security, Risk and Trust and 2011 IEEE Third Int'l Conference on Social Computing, pp. 180-185. IEEE (2011). DOI 10.1109/PASSAT/SocialCom.2011.26

54. Quijano-Sanchez, L., Recio-Garcia, J.a., Diaz-Agudo, B.: Personality and Social Trust in Group Recommendations. 2010 22nd IEEE International Conference on Tools with Artificial Intelligence (c), 121-126 (2010). DOI 10.1109/ICTAI.2010.92

55. Rawlings, D., Ciancarelli, V.: Music Preference and the Five-Factor Model of the NEO Personality Inventory. Psychology of Music 25(2), 120-132 (1997). DOI 10.1177/ 0305735697252003

56. Recio-Garcia, J.A., Jimenez-Diaz, G., Sanchez-Ruiz, A.A., Diaz-Agudo, B.: Personality aware recommendations to groups. In: Proceedings of the third ACM conference on Recommender systems - RecSys '09, p. 325. ACM Press, New York, New York, USA (2009). DOI 10.1145/1639714.1639779

57. Rentfrow, P.J., Goldberg, L.R., Zilca, R.: Listening, watching, and reading: the structure and correlates of entertainment preferences. Journal of personality 79(2), 223-58 (2011). DOI 10.1111/j.1467-6494.2010.00662.x

58. Rentfrow, P.J., Gosling, S.D.: The do re mi's of everyday life: The structure and personality correlates of music preferences. Journal of Personality and Social Psychology 84(6), 12361256 (2003). DOI 10.1037/0022-3514.84.6.1236 
59. Ross, C., Orr, E.S., Sisic, M., Arseneault, J.M., Simmering, M.G., Orr, R.R.: Personality and motivations associated with facebook use. Computers in Human Behavior 25(2), 578-586 (2009)

60. Schrammel, J., Köffel, C., Tscheligi, M.: Personality traits, usage patterns and information disclosure in online communities. Proceedings of the 23rd British HCI ...pp. 169-174 (2009)

61. Selfhout, M., Burk, W., Branje, S., Denissen, J., van Aken, M., Meeus, W.: Emerging late adolescent friendship networks and Big Five personality traits: a social network approach. Journal of personality 78(2), 509-38 (2010). DOI 10.1111/j.1467-6494.2010.00625.x

62. Sha, X., Quercia, D., Michiardi, P., Dell'Amico, M.: Spotting trends. In: Proceedings of the sixth ACM conference on Recommender systems - RecSys '12, p. 51. ACM Press, New York, New York, USA (2012). DOI 10.1145/2365952.2365967

63. Shen, J., Brdiczka, O., Liu, J.: Understanding Email Writers: Personality Prediction from Email Messages. User Modeling, Adaptation, and Personalization pp. 318-330 (2013). DOI 10.1007/978-3-642-38844-6\_29

64. Soloman, B.A., Felder, R.M.: Index of Learning Styles Questionnaire (2014). URL http: //www.engr.ncsu.edu/learningstyles/ilsweb.html

65. Stewart, B.: Personality And Play Styles: A Unified Model (2011)

66. Thomas, K.W.: Conflict and conflict management: Reflections and update. Journal of Organizational Behavior 13(3), 265-274 (1992). DOI 10.1002/job.4030130307

67. Tintarev, N., Dennis, M., Masthoff, J.: Adapting Recommendation Diversity to Openness to Experience: A Study of Human Behaviour. User Modeling, Adaptation, and Personalization, Lecture Notes in Computer Science Volume 7899 (I), 190-202 (2013). DOI 10.1007/978-3-642-38844-6\_16

68. Tiroshi, A., Kuflik, T.: Domain ranking for cross domain collaborative filtering. User Modeling, Adaptation, and Personalization pp. 328-333 (2012). DOI 10.1007/978-3-642-31454-4\ 30

69. Tkalcic, M., Kunaver, M., Košir, A., Tasic, J.: Addressing the new user problem with a personality based user similarity measure. Joint Proceedings of the Workshop on Decision Making and Recommendation Acceptance Issues in Recommender Systems (DEMRA 2011) and the 2nd Workshop on User Models for Motivational Systems: The affective and the rational routes to persuasion (UMMS 2011) (2011)

70. Tkalčič, M., Burnik, U., Košir, A.: Using affective parameters in a content-based recommender system for images. User Modeling and User-Adapted Interaction 20(4), 279-311 (2010). DOI 10.1007/s11257-010-9079-Z

71. Tkalčič, M., Košir, A., Tasič, J.: The LDOS-PerAff-1 corpus of facial-expression video clips with affective, personality and user-interaction metadata. Journal on Multimodal User Interfaces 7(1-2), 143-155 (2013). DOI 10.1007/s12193-012-0107-7

72. Tkalčič, M., Kunaver, M., Tasič, J., Košir, A.: Personality Based User Similarity Measure for a Collaborative Recommender System. 5th Workshop on Emotion in Human-Computer Interaction-Real World Challenges p. 30 (2009)

73. Winoto, P., Tang, T.: If You Like the Devil Wears Prada the Book, Will You also Enjoy the Devil Wears Prada the Movie? A Study of Cross-Domain Recommendations. New Generation Computing 26(3), 209-225 (2008). DOI 10.1007/s00354-008-0041-0

74. Wu, W., Chen, L., He, L.: Using personality to adjust diversity in recommender systems. Proceedings of the 24th ACM Conference on Hypertext and Social Media - HT '13 (May), 225-229 (2013). DOI 10.1145/2481492.2481521

75. Ziegler, C.N., McNee, S.M., Konstan, J.A., Lausen, G.: Improving recommendation lists through topic diversification. In: Proceedings of the 14th International Conference on World Wide Web, WWW '05, pp. 22-32. ACM, New York, NY, USA (2005). DOI 10.1145/1060745.1060754 